\title{
Modulating NAD+ metabolism to prevent acute kidney injury
}

\author{
Heerajnarain Bulluck $\mathrm{PhD}^{1}$, Derek J Hausenloy $\mathrm{PhD}^{2-7}$
}

${ }^{1}$ Norfolk and Norwich University Hospital, UK

${ }^{2}$ The Hatter Cardiovascular Institute, University College London, London, UK

3 The National Institute of Health Research University College London Hospitals

Biomedical Research Centre, London, UK

${ }^{4}$ National Heart Centre Singapore, Singapore

${ }^{5}$ Barts Heart Centre, St Bartholomew's Hospital, London, UK

${ }^{6}$ Cardiovascular and Metabolic Disorders Program, Duke-National University of Singapore Medical School, Singapore

${ }^{7}$ Yong Loo Lin School of Medicine, National University Singapore, Singapore

Competing interests: None

Funding: DJH was supported by the British Heart Foundation (FS/10/039/28270), the National Institute for Health Research University College London Hospitals Biomedical Research Centre, Duke-National University Singapore Medical School, Singapore Ministry of Health's National Medical Research Council under its Clinician ScientistSenior Investigator scheme (NMRC/CSA-SI/0011/2017) and Collaborative Centre Grant scheme (NMRC/CGAug16C006), and the Singapore Ministry of Education Academic Research Fund Tier 2 (MOE2016-T2-2-021). This article is based upon work from COST Action EU-CARDIOPROTECTION CA16225 supported by COST (European Cooperation in Science and Technology).

\section{Corresponding author:}

Professor Derek J Hausenloy

Cardiovascular \& Metabolic Disorders Program

Duke-NUS Medical School Singapore

8 College Road,

Singapore 169857

Tel +65 65166719

Email derek.hausenloy@duke-nus.edu.sg 
Impaired de novo NAD+ biosynthesis predisposes to acute kidney injury, and augmenting $\mathrm{NAD}^{+}$metabolism with oral nicotinamide supplementation may prevent acute kidney injury.

Over one fifth of all hospitalized patients develop acute kidney injury (AKI), a condition which has been defined as an abrupt decline in renal function. The incidence of $A K I$ is highest in intensive care unit (ICU) patients (32\%), and in patients undergoing cardiac surgery (24\%). ${ }^{1}$ AKI-associated all-cause mortality occurs in $23 \%$ of hospitalized patients, and depending on its severity, AKI confers 3-7x increased risk of death. ${ }^{1} \mathrm{AKI}$ that is severe enough to require renal replacement therapy (RRT, dialysis or kidney transplantation) occurs in $2.3 \%$ of all hospitalized patients, increasing the risk of death by 24 -fold. ${ }^{1}$ As there is currently no effective therapy for preventing $\mathrm{AKI}$, there is an urgent unmet medical need to discover novel renoprotective therapies for improving clinical outcomes in AKI patients.

Nicotinamide adenine dinucleotide $\left(\mathrm{NAD}^{+}\right)$is a universal electron acceptor that plays an essential role in cellular energy metabolism, and adaptive stress responses. ${ }^{2}$ Its depletion has been recognized to be a fundamental feature of aging that predisposes to a wide range of age-related neurodegenerative, ${ }^{2}$ cardiac and renal diseases. ${ }^{3}$ In this issue of Nature Medicine, Mehr et al ${ }^{4}$ identify that impaired de novo NAD ${ }^{+}$biosynthesis at quinolinate phosphoribosyltransferase (QPRT) predisposes to AKI, and results in elevated levels of urinary quinolinate/tryptophan $(\mathrm{uQ} / \mathrm{T})$, a potential novel biomarker for AKI. ${ }^{4}$ Furthermore, they demonstrate that oral nicotinamide supplementation, to augment renal $\mathrm{NAD}^{+}$metabolism, was able to prevent $\mathrm{AKI} .{ }^{4}$

The authors first carried out an unbiased metabolomics screen of 204 urinary metabolites in a mouse model of $\mathrm{AKI}$ and identified elevated levels of urinary quinolinate, an intermediate in the de novo $\mathrm{NAD}^{+}$biosynthetic pathway from 
tryptophan. Transient renal ischemia, which induces AKI by causing glomerular injury, was shown in kidney tissue to reduce $\mathrm{NAD}^{+}$content, increase quinolinate levels, and decrease gene expression of QPRT (the enzyme which converts quinolinate to nicotinic acid mononucleotide, a precursor of $N A D^{+}$).

The authors further confirmed the role of QPRT deficiency in the development of AKI by showing that mice partially deficient in QPRT have elevated levels of urinary quinolinate under baseline conditions, even when this was normalized to urinary tryptophan levels (expressed as $u Q / T$ ), and were found to be more susceptible to AKI following transient renal ischemia. Interestingly, administration of nicotinamide (a precursor of $\mathrm{NAD}^{+}$) prior to renal ischemia made them less prone to develop $\mathrm{AKI}$, suggesting that augmentation of renal $\mathrm{NAD}^{+}$metabolism was renoprotective.

Next, the authors investigated whether impaired de novo NAD+ biosynthesis linked to QPRT also occurs during transient renal ischemia in adult patients undergoing cardiac surgery. AKI occurs in $24 \%$ of adult patients undergoing cardiac surgery, ${ }^{1}$ with modest rises in post-operative serum creatinine being associated with higher 30-day mortality, ${ }^{5,6}$ and AKI patients requiring RRT having in-hospital mortality rates as high as $60 \% .^{7}$ In a small cohort of 6 patients undergoing cardiac surgery on cardiopulmonary bypass who developed post-operative $\mathrm{AKI}$, the authors demonstrated that $\mathrm{uQ} / \mathrm{T}$ increased significantly at 24 hours and persisted for 5 days following surgery, when compared to 6 patients who underwent cardiac surgery but did not develop AKI. In a separate large prospective study of 215 ICU patients, the authors found that elevations in urinary quinolinate and $\mathrm{uQ} / \mathrm{T}$ occurred at a median of 2.5 days prior to the diagnosis of $\mathrm{AKI}$. Importantly, levels of $\mathrm{uQ} / \mathrm{T}$ were able to identify patients at risk of developing $\mathrm{AKI}$, and predict adverse clinical outcomes (severe AKI, RRT- and AKI-related death, and in-hospital death). These findings suggest that a deficient QPRT may contribute to the development of $A K I$ in humans, and that $u Q / T$ 
may be a novel biomarker for detection of $\mathrm{AKI}$ and for prediction of adverse outcomes in ICU patients.

The authors next tested the safety and tolerability of oral nicotinamide administration in human volunteers. A 3-day course of high dose (3gm/day) oral nicotinamide was well-tolerated, led to detectable levels of circulating nicotinamide, and was not nephrotoxic (as it did not affect kidney function) in a small group of healthy volunteers $(n=8)$. They went on to carry out a Phase 1 randomized controlled trial of oral nicotinamide versus placebo in adults undergoing cardiac surgery to assess its safety and tolerability. The study comprised 3 treatment groups: nicotinamide at two doses and placebo $(n=14)$, administered orally or via a nasogastric tube on days $-1,0$, and +1 the day before, the day of and the day after surgery. Blood and urine nicotinamide levels were significantly higher in patients receiving nicotinamide, and the side-effect profile was no different to placebo. Although this study was not designed to test efficacy of nicotinamide, the latter did significantly reduce post-operative levels of serum cardiac troponin-T and serum creatinine levels over the 3-day post-operative period, suggesting a potential cardioprotective and renoprotective effect.

The findings from the study by Mehr et al ${ }^{4}$ suggest that reduced levels of kidney NAD ${ }^{+}$ due to impaired de novo NAD+ biosynthesis at QPRT predisposes to AKI (Figure 1). Further studies are needed to elucidate the mechanisms through which kidney levels of QPRT are reduced following the acute insult. uQ/T may be a novel biomarker for identification of patients at risk of $\mathrm{AKI}$, and it may also predict adverse clinical outcomes in ICU patients. Further work is needed to assess the robustness of $u Q / T$ as a biomarker for early detection of AKI and for predicting adverse clinical outcomes, and to investigate how its performance as a biomarker compares with other AKI biomarkers such as neutrophil gelatinase-associated lipocalin. ${ }^{8}$ Finally, administration of oral nicotinamide, to augment renal $\mathrm{NAD}^{+}$metabolism, is safe and tolerable in cardiac surgery patients, and has the therapeutic potential to be renoprotective. Whether, 
nicotinamide can prevent $\mathrm{AKI}$ and improve clinical outcomes in cardiac surgery and ICU patients, needs to be investigated in large adequately powered randomized clinical trials. Nicotinamide may be a promising and low-cost therapy for not only preventing $\mathrm{AKI}$, as it may also have the therapeutic potential to protect other organs against acute injury such as the heart (in acute myocardial infarction), brain (following stroke) and liver (during hepatic surgery). 


\section{References:}

1. Susantitaphong, P., et al. World incidence of AKI: a meta-analysis. Clinical journal of the American Society of Nephrology : CJASN 8, 1482-1493 (2013).

2. Fang, E.F., et al. NAD(+) in Aging: Molecular Mechanisms and Translational Implications. Trends Mol Med 23, 899-916 (2017).

3. Hershberger, K.A., Martin, A.S. \& Hirschey, M.D. Role of $N A D(+)$ and mitochondrial sirtuins in cardiac and renal diseases. Nat Rev Nephrol 13, 213225 (2017).

4. Mehr, A.P., et al. De novo NAD+ biosynthetic impairment in acute kidney injury in humans. Nature medicine X, XXX (2018).

5. Lassnigg, A., et al. Minimal changes of serum creatinine predict prognosis in patients after cardiothoracic surgery: A prospective cohort study. Journal of the American Society of Nephrology 15, 1597-1605 (2004).

6. Lok, C.E., Austin, P.C., Wang, H. \& Tu, J.V. Impact of renal insufficiency on short- and long-term outcomes after cardiac surgery. American Heart Journal 148, 430-438 (2004).

7. Bhatt, G.C. \& Das, R.R. Early versus late initiation of renal replacement therapy in patients with acute kidney injury-a systematic review \& meta-analysis of randomized controlled trials. BMC Nephrol 18, 78 (2017).

8. Bulluck, H., et al. Neutrophil gelatinase-associated lipocalin prior to cardiac surgery predicts acute kidney injury and mortality. Heart, 313-317 (2017). 
Figure 1: The study by Mehr et al. ${ }^{4}$ provides 3 key findings for patients at risk of developing acute kidney injury (AKI). The authors have uncovered a new mechanism underlying $A K I$, by identifying in kidneys subjected to transient ischemia that reduced levels of quinolinate phosphoribosyltransferase (QPRT) result in impaired renal de novo biosynthesis of $\mathrm{NAD}^{+}$, and predispose to AKI. This metabolic defect increased levels of urinary quinolate/tryptophan, providing a potential novel biomarker for identifying patients at risk of $\mathrm{AKI}$, and for predicting adverse clinical outcomes. Finally, the authors have demonstrated that augmenting renal $\mathrm{NAD}^{+}$metabolism with oral nicotinamide (a precursor of $\mathrm{NAD}^{+}$) could prevent $\mathrm{AKI}$, providing a potential novel therapy for preventing AKI and improving clinical outcomes in patients admitted to ICU or undergoing cardiac surgery. 